\title{
PULMONARY ALVEOLAR PROTEINOSIS
}

natuire DISEASE

REVIEWS PRIMERS

Pulmonary alveolar proteinosis

(PAP) is a syndrome characterized by abnormal accumulation of alveolar surfactant in the lungs and dysfunction of the alveolar macrophages. PAP can be classified as primary (autoimmune or hereditary), secondary or congenital, depending on the underlying mechanism.

\section{EPIDEMIOLOGY}

The overall prevalence of PAP has been estimated to be at least 7 per million individuals in the

general population in the United States and Japan, where the largest population studies have been conducted. However, the actual prevalence is probably higher, as PAP often remains undiagnosed or misdiagnosed for a long time. Autoimmune PAP accounts for $\sim 90 \%$ of all patients.

\section{$\triangle \triangle$ QUALITY OF LIFE}

The quality of life varies widely among patients depending on the cause, clinical course and disease severity; most patients have exertional dyspnoea and frequent cough. Granulocyte-macrophage colony-stimulating factor (GM-CSF) augmentation therapy seems to improve quality of life and dyspnoea. Remission has been reported, but it remains unclear whether these instances represent true disease-free remission or subclinical disease.

$$
\begin{aligned}
& \text { e. Autoimmune PAP } \\
& \text { ts. }
\end{aligned}
$$

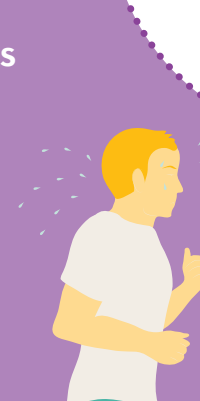

\section{(1)}

(D)

$\because$

$$
\begin{gathered}
\text { efflux } \\
\text { cholesterol }
\end{gathered}
$$

macrophages

depend on

GM-CSF signalling

for maintaining

efflux

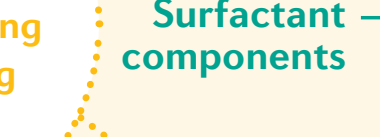

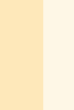

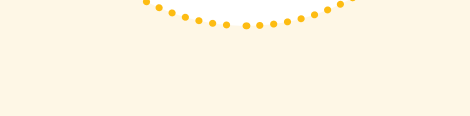

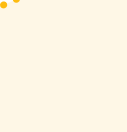

GM-CSF

GM-C

$.00 \cdots \cdots \cdots$ Autoimmune PAP is characterized by elevated levels of neutralizing GM-CSF autoantibodies
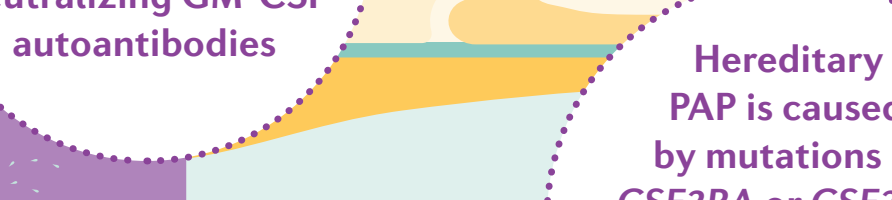

PAP is caused

by mutations in

CSF2RA or CSF2RB,

encoding the two

subunits of the

GM-CSF receptor

1340 38

accumulates in intracellular

lipid droplets, which lead

to the formation of

foamy macrophages

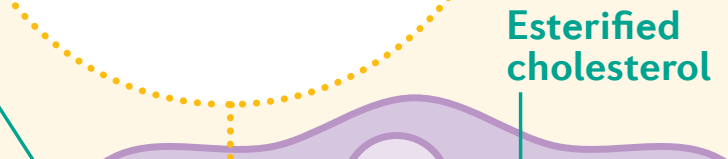

(e) 00

0

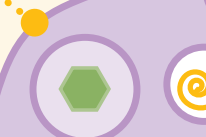

(e)

(1)

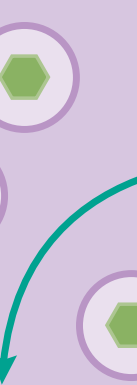

-

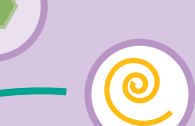

(2)
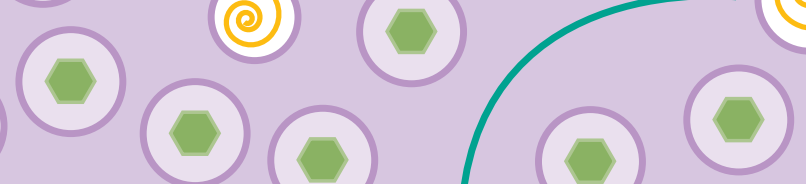

(อ)
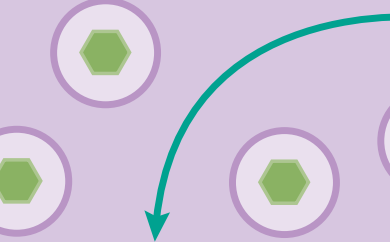

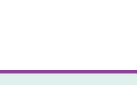

